\title{
A Mathematical Model to Study the Human Brain Information Processing Dynamics
}

\author{
Shikaa Samuel ${ }^{1, *}$, Taparki Richard ${ }^{1}$, Ajai John Tyavbee², Aboiyar Terhemen ${ }^{3}$ \\ ${ }^{1}$ Department of Mathematical Sciences, Taraba State University, Jalingo, Nigeria \\ ${ }^{2}$ Department of Science Education, Taraba State University, Jalingo, Nigeria \\ ${ }^{3}$ Department of Mathematics, Statistics and Computer Science, University of Agriculture, Makurdi, Nigeria \\ Email address: \\ shiksmen@gmail.com (S. Samuel), richardtaparki@gmail.com (T. Richard), jtajai@gmail.com (A. J. Tyavbee) \\ t_aboiyar@yahoo.co.uk (A. Terhemen)
}

\section{To cite this article:}

Shikaa Samuel, Taparki Richard, Ajai John Tyavbee, Aboiyar Terhemen. A Mathematical Model to Study the Human Brain Information Processing Dynamics. American Journal of Applied Mathematics. Vol. 3, No. 5, 2015, pp. 233-242. doi: 10.11648/j.ajam.20150305.15

\begin{abstract}
This research work studies the human brain information processing dynamics by transforming the stage model formulated by Atkinson and Shiffrin into two deterministic mathematical models. This makes it more amenable to mathematical analysis. The two models are bottom-up processing mathematical model and top-down processing mathematical model. The bottom-up processing is data driven while the top-down processing is triggered by experience or prior knowledge. Both analytical and numerical methods are used in the analysis of the models. The existence and stability of equilibrium states of the models are investigated, and threshold values of certain parameters of the models arising from the investigation were obtained and interpreted in physical terms. Numerical experiments are also carried out using hypothetical data to further investigate the effect of certain parameters on the human brain information processing process. The results show that attention, repetition and rehearsal play significant roles in learning process. Furthermore, repetition and rehearsal is strongly recommended as an effective way of retaining information. In addition, the instructors should ensure that the students feel physically and psychologically safe in any environment in order to pay adequate attention.
\end{abstract}

Keywords: Brain, Information Processing, Bottom-Up, Top-Down, Attention, Automaticity

\section{Introduction}

In humans and other animals, sense organs are faculties by which outside information is received for evaluation and response. This is accomplished by the effect of a particular stimulus on a specialized organ (eye, ear, etc.), which then transmits impulses to the brain via a nerve or nerves.

[12] Explains the structure of a brain cell, called a neuron, and describes how stimuli travelling down the axon of a neuron release neurotransmitters that jump across a tiny gap between neurons called a synapse. Memories are formed when a group of neurons is stimulated to form a pattern of responses. Just as muscles improve with exercise, the brain seems to improve with use. While learning does not increase the number of brain cells, it does increase their size, their branches, and their ability to form more complex networks. The brain is wired to notice events that affect survival first, events that affect emotions second, and events that contribute to learning, third. From this hierarchy, it is clear that someone must feel physically and psychologically safe in any environment in order to pay attention.

We will present and analyse two deterministic mathematical models from the stage model developed by [2] that is made up of three compartments, namely: sensory memory (SSM), short-term memory (STM) and long-term memory (LTM).

The belief in the interaction of new information with stored information is a third key point of cognitive study. This is usually demonstrated with a bottom-up or top-down system or a combination of the two. A bottom-up system is predicated on the belief that new information is seen as an initiator, which the brain attempts to match with existing concepts in order to break down characteristics or defining attributes [5]. A top-down system suggests an opposite approach. The existing information is the initiator and memory representations are evaluated, and then matched to the stimuli [10].

Sensory memory represents the initial stage of stimuli perception. It is associated with the senses, and there seems 
to be a separate section for each type of sensual perception, each with its own limitations and devices. The transfer of new information quickly to the next stage of processing is of critical importance. This stage of memory is temporally limited which means that information stored here begins to decay rapidly if not transferred to the next stage. This occurs in as little as $1 / 2$ second for visual stimuli and three seconds for auditory stimuli. To this end, attention and automaticity are the two major influences on the sensory [7].

The second stage of information processing is the working or short-term memory. This stage is often viewed as active or conscious memory because it is the part of memory that is being actively processed while new information is being taken in. Short-term memory has a very limited capacity and unrehearsed information will begin to be lost from it within 15-30 seconds if other action is not taken. There are two main ways that are effective in processing information while it is in short-term memory. Rote or maintenance rehearsal is the first but less desirable of these methods. This type of rehearsal is intended only to keep information until it can be processed further. It consists mainly of some sort of repetition of the new information, and if it is not processed further will be lost memory [13].

Like short-term memory, long-term memory houses all previous perceptions, knowledge, and information learned by an individual, but it is not a static file system that is used only for information retrieval. According to [1], the long-term memory is that more permanent store in which information can reside in a dormant state - out of mind and unused - until you fetch it back into consciousness. In order to incorporate new information, long-term memory must be in communication with short-term memory and must be dynamic. There are several categories of long-term memory, and there are many suggestions as to how memory units are represented in the mind.

Attention is defined by [14] as the "limitations in our perceptual processing and response generation: to attend to one of this is to not attend to others". Attention does facilitate the integration and transfer of the information being attended, but it is impacted by many factors including the meaningfulness of the new stimulus to the learner, the similarity between competing ideas or stimuli, the complexity of the new information, and the physical ability of the person to attend.

Automaticity is almost the exact opposite of attention. According to [3], "When tasks are over learned or sources of information become habitual, to the extent that their attention requirements are minimal, automaticity has occurred". Automaticity allows attention to be redirected to other information or stimuli and allows for the ability of multitasking without distracting totally from the acquisition of new information. Several researchers have worked on human brain information processing. Particularly, [16] examine the top-down and bottom-up processes in speech comprehension while [8] commented on top-down verses bottom-up control of attention in the prefrontal and posterior parietal cortices.

[11] proposed that under normal viewing conditions, the main processes of feature binding and perception proceed largely independently of top-down selective attention. The model presented by [15] makes a strong prediction that there is no way to independently store the same or very similar points on a feature dimension (they will merge). The authors interpret a recent paper examining the influence of feature similarity on memory performance ([9]) as evidence for their merging account, proposing that where two very similar features are stored, the representation of these features will be roughly their average value.

In this study, we formulate two compartmental deterministic mathematical models to study the human brain information processing dynamics using the stage model developed by [2]; and considering the bottom-up and topdown processing systems separately.

\section{The Stage Model}

The stage model for human brain information processing dynamics developed by [2] hypothesizes that, as new information is taken in; it is in some way manipulated before it is stored. The stage theory model recognizes three types or stages of memory: sensory memory, short-term or working memory, and long-term memory. The sensory memory acts as a portal for all information that is to become part of memory. Attention and automaticity are the two major effects on sensory memory. When tasks are over learned or sources of information become habitual, to the extent that their attention requirements are minimal, automaticity has occurred. Automaticity allows attention to be forwarded to other information or stimuli. The diagram is given below:

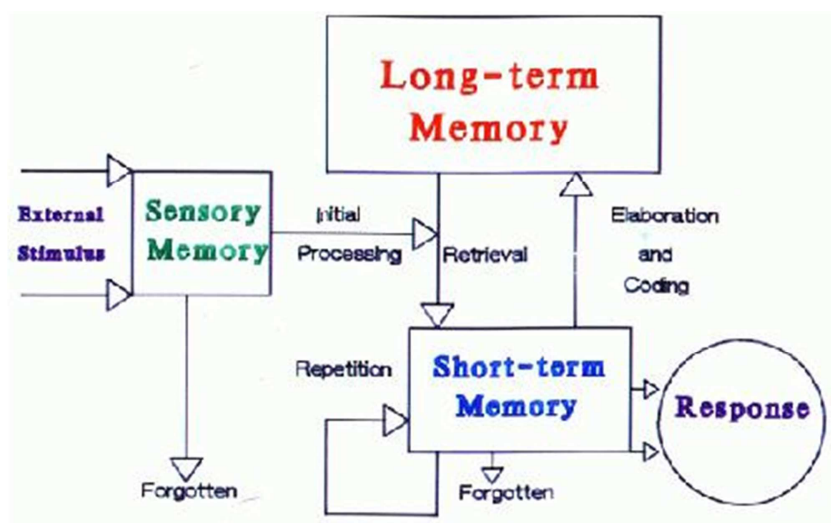

Figure 1. A comprehensive version of [2] stage model as presented by [7].

\section{Formulation of the Two Mathematical Models}

The bottom-up and top-down processing models are presented in this section. The two models assume that: The brain under observation is naturally healthy. The encoding and storage systems are either bottom-up or top-down processing but not both. All the compartments have unlimited storage capacity. The model is age independent. 
Table 1. State variables, parameters and descriptions.

\begin{tabular}{|c|c|}
\hline$x_{1}(t):$ & $\begin{array}{l}\text { The amount of information in the Sensory Memory at time } t \\
\text { (bottom-up processing). }\end{array}$ \\
\hline$y_{1}(t):$ & $\begin{array}{l}\text { The amount of information in the Short-Term Memory at time } t \\
\text { (bottom-up processing). }\end{array}$ \\
\hline$z_{1}(\mathrm{t}):$ & $\begin{array}{l}\text { The amount of information in the Long-Term Memory at time } t \\
\text { (bottom-up processing) }\end{array}$ \\
\hline$x_{2}(t):$ & $\begin{array}{l}\text { The amount of information in the Sensory Memory at time } t \\
\text { (top-down processing). }\end{array}$ \\
\hline$y_{2}(t):$ & $\begin{array}{l}\text { The amount of information in the Short-Term Memory at time } t \\
\text { (top-down processing). }\end{array}$ \\
\hline$z_{2}(t):$ & $\begin{array}{l}\text { The amount of information in the Long-Term Memory at time } t \\
\text { (top-down processing). }\end{array}$ \\
\hline$I(t):$ & $\begin{array}{l}\text { The rate of inflow of stimuli into the sensory memory (brain) at } \\
\text { time } t\end{array}$ \\
\hline$\tau:$ & Forgetfulness coefficient \\
\hline$\alpha_{1}:$ & Attention coefficient for bottom-up processing \\
\hline$\alpha_{2}:$ & Attention coefficient for top-down processing. \\
\hline$\beta:$ & Rehearsal coefficient \\
\hline$\rho:$ & Repetition coefficient. \\
\hline$\mu:$ & Retrieval coefficient \\
\hline$\kappa:$ & Arbitrary constant \\
\hline$v:$ & Automaticity coefficient. \\
\hline$\omega:$ & Response coefficient. \\
\hline$t:$ & Time \\
\hline
\end{tabular}

The schematic diagrams for the two mathematical models are presented in Figure (2) and (3) below:

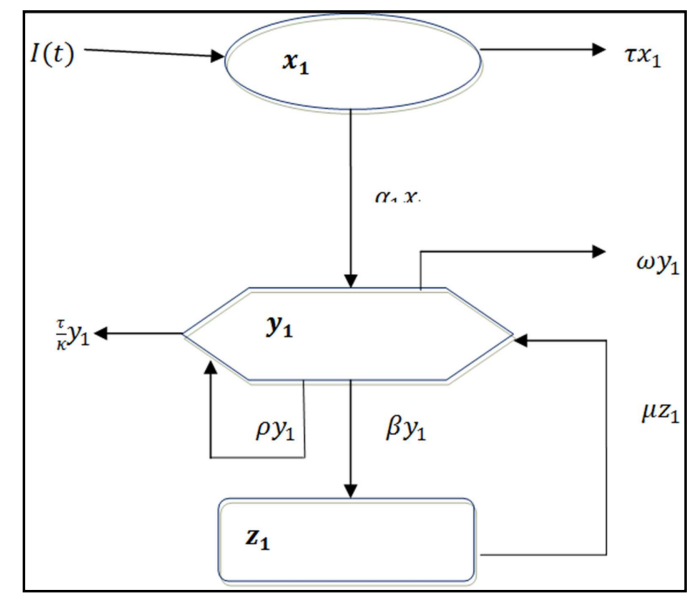

Figure 2. Flow Diagram of human brain information processing dynamics (bottom-up model).

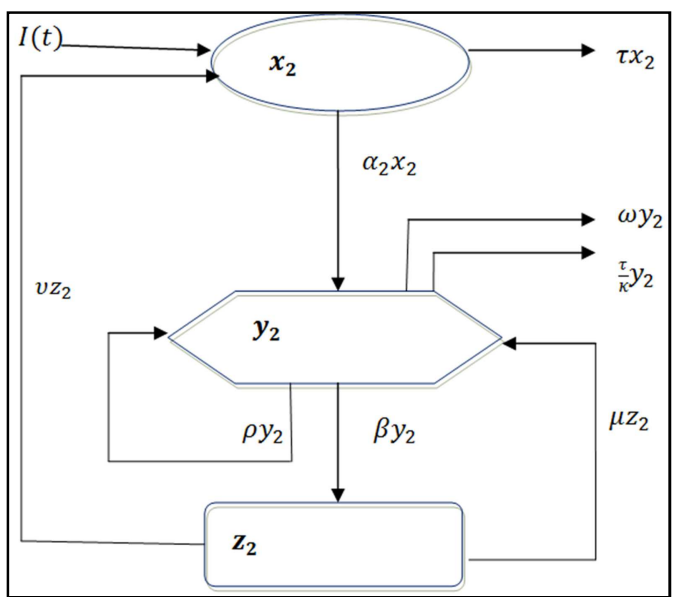

Figure 3. Flow Diagram of human brain information processing dynamics (top-down model).

\subsection{Bottom-Up Processing Mathematical Model}

Environmental information is processed by sensory registers in the sensory memory (SSM) and entered into short-term memory (STM) when attention is given to it. The information remains temporarily in STM, the length of stay depending on repetition and rehearsal processes. While information remains in STM it may be translated into longterm memory (LTM) by maintained rehearsal. The information stored in LTM will be used only if it is retrieved back into the STM information. The phenomenon is represented by the following equations:

$$
\begin{gathered}
\frac{d x_{1}}{d t}=I(t)-\left(\alpha_{1}+\tau\right) x_{1} \\
\frac{d y_{1}}{d t}=\alpha_{1} x_{1}+\left(\rho-\beta-\omega-\frac{\tau}{\kappa}\right) y_{1}+\mu z_{1} \\
\frac{d z_{1}}{d t}=\beta y_{1}-\mu z_{1} \\
x_{1}(0)=0, y_{1}(0)=0, z_{1}(0)=0, \\
\alpha_{1}, \beta, \mu, \tau, \rho, \omega, \nu \geq 0, \tau<\kappa
\end{gathered}
$$

\subsection{Top-Down Processing Mathematical Model}

Here, the environmental information that is processed into the sensory memory triggers pre-existed information of a similar sort in the long-term memory (LTM) via a process called automaticity which cause less attention to be given to the piece of information thereby causing a lesser percentage of the environmental information to be incorporated into the short-term and long-term memories respectively. In this model also, the stored information must be retrieved from the long-term memory before use. The following equations represent the process:

$$
\begin{gathered}
\frac{d x_{2}}{d t}=I(t)-\left(\alpha_{2}+\tau\right) x_{2}+v z_{2} \\
\frac{d y_{2}}{d t}=\alpha_{2} x_{2}+\left(\rho-\beta-\omega-\frac{\tau}{\kappa}\right) y_{2}+\mu z_{2} \\
\frac{d z_{2}}{d t}=\beta y_{2}-(\mu+v) z_{2} \\
x_{2}(0)=0, y_{2}(0)=0, z_{2}(0)=350, \\
\alpha_{2}, \beta, \mu, \tau, \rho, \omega, \nu \geq 0, \tau<\kappa .
\end{gathered}
$$

\subsection{Analysis of the Two Mathematical Models}

The following theorems will be found useful in this section:

\subsubsection{Theorem 3.1 [6]}

Consider the linear system $y^{\prime}=A(t) y+b(t)$. Suppose that the matrix $A$ is a constant matrix, with eigenvalues $\lambda_{1}, \lambda_{2}, \ldots, \lambda_{\mathrm{n}}$ and $\operatorname{Re}\left(\lambda_{i}\right)<0$ for all $i=1,2, \ldots, n$. Let $b(t)$ be defined and continuous for all $t \geq t_{0}$ and satisfy $\frac{\|b(t)\|}{|t|} \rightarrow$ 0 as $t \rightarrow \infty$, then the equilibrium state of $y^{\prime}=A(t) y+b(t)$ is uniformly and asymptotically stable whenever it exists.

\subsubsection{Theorem 3.2 (Routh- Hurwitz Criterion) [4]}

Let $P(\lambda)=\lambda^{3}+A_{2} \lambda^{2}+A_{1} \lambda+A_{0}=0$ be the 
characteristic equation in $\lambda$ corresponding to a 3-dimensional linear system, where $A_{0}, A_{1}$ and $A_{2}$ are constants. Then, the eigenvalues $\lambda_{1}, \lambda_{2}$ and $\lambda_{3}$ have negative real parts if and only if

(i) $A_{0}, A_{1}, A_{2}>0$, and

(ii) $A_{2} A_{1}>A_{0}$.

\subsubsection{Existence of the Equilibrium State of the Model Equations of Bottom-up and Top-Down Information Processing Dynamics}

The existence of the equilibrium points $\left(\mathrm{x}_{1}^{*}, \mathrm{y}_{1}^{*}, \mathrm{z}_{1}^{*}\right)$ and $\left(\mathrm{x}_{2}^{*}, \mathrm{y}_{2}^{*}, \mathrm{z}_{2}^{*}\right)$ is subject to $\mathrm{x}_{1}^{*}>0, \mathrm{y}_{1}^{*}>0, \mathrm{z}_{1}^{*}>0, \mathrm{x}_{2}^{*}>0, \mathrm{y}_{2}^{*}>$ $0, \mathrm{z}_{2}^{*}>0$ and $\mathrm{I}^{*}>0$. We equate the left hand sides (LHS) of the model equations (3.1)-(3.3) to zero and solving them simultaneously we obtain the only equilibrium state of the bottom-up model as:

$$
\left.\begin{array}{c}
\mathrm{X}_{1}^{*}=\frac{\mathrm{I}^{*}}{\left(\alpha_{1}+\tau\right)} \\
\mathrm{y}_{1}^{*}=\frac{-\alpha_{1} \mathrm{I}^{*}}{\left(\alpha_{1}+\tau\right)\left(\rho-\omega-\frac{\tau}{\kappa}\right)} \\
\mathrm{z}_{1}^{*}=\frac{-\alpha_{1} \beta \mathrm{I}^{*}}{\mu\left(\alpha_{1}+\tau\right)\left(\rho-\omega-\frac{\tau}{\kappa}\right)}
\end{array}\right\}
$$

provided

$$
\rho<\omega+\frac{\tau}{\kappa} \cdot(3.7)^{*}
$$

Similarly, we obtain the only equilibrium state of the topdown model as

$$
\left.\begin{array}{l}
x_{2}^{*}=\frac{\left[(\mu+v)\left(\rho-\beta-\omega-\frac{\tau}{\kappa}\right)+\beta \mu\right] I^{*}}{(\mu+v)\left(\alpha_{2}+\tau\right)\left(\rho-\beta-\omega-\frac{\tau}{\kappa}\right)+\beta\left[\mu\left(\alpha_{2}+\tau\right)+\alpha_{2} v\right]} \\
y_{2}^{*}=\frac{-\alpha_{2}(\mu+v) I^{*}}{(\mu+v)\left(\alpha_{2}+\tau\right)\left(\rho-\beta-\omega-\frac{\tau}{\kappa}\right)+\beta\left[\mu\left(\alpha_{2}+\tau\right)+\alpha_{2} v\right]} \\
z_{2}^{*}=\frac{-\alpha_{2} \beta I^{*}}{(\mu+v)\left(\alpha_{2}+\tau\right)\left(\rho-\beta-\omega-\frac{\tau}{\kappa}\right)+\beta\left[\mu\left(\alpha_{2}+\tau\right)+\alpha_{2} v\right]}
\end{array}\right\}
$$

provided

$$
\rho+\frac{\beta \mu}{\mu+v}<\beta+\omega+\frac{\tau}{\kappa}
$$

\subsubsection{Stability Analysis of the Equilibrium State of the \\ Model (Bottom-Up Processing)}

The stability analysis of the equilibrium state of the bottom-up processing model is stated and proved in the follow theorem below:

\subsubsection{Theorem 3.3}

Given $\alpha_{1}, \beta, \mu, \tau, \rho, \omega, \nu \geq 0, \tau<\kappa$. If $\rho-\omega-\frac{\tau}{\kappa}<0$ and $\mathrm{I}^{*} \neq 0$, then the equilibrium state $E_{1}=\left(\frac{I^{*}}{\left(\alpha_{1}+\tau\right)}, \frac{-\alpha_{1} I^{*}}{\left(\alpha_{1}+\tau\right)\left(\rho-\omega-\frac{\tau}{\kappa}\right)}, \frac{-\alpha_{1} \beta I^{*}}{\mu\left(\alpha_{1}+\tau\right)\left(\rho-\omega-\frac{\tau}{\kappa}\right)}\right)$ of the system (3.1)-(3.3) is uniformly and asymptotically stable UAS.

Proof:

The system (3.1)-(3.3) is nonhomogeneous linear systems with constant coefficients, which can be written in matrixvector form as

$$
y^{\prime}=A y+b(\mathrm{t})
$$

where

$$
\begin{gathered}
y=\left(\begin{array}{l}
x_{1} \\
y_{1} \\
z_{1}
\end{array}\right), \\
A=\left(\begin{array}{ccc}
-\left(\alpha_{1}+\tau\right) & 0 & 0 \\
\alpha & \left(\rho-\beta-\omega-\frac{\tau}{\kappa}\right) & \mu \\
0 & \beta & -\mu
\end{array}\right),
\end{gathered}
$$

and

$$
b(t)=\left(\begin{array}{c}
I(t) \\
0 \\
0
\end{array}\right)
$$

Applying theorem 3.1 above, we have

$$
\begin{gathered}
\therefore|\mathrm{A}-\lambda \mathrm{I}|= \\
\left|\begin{array}{ccc}
-\left(\alpha_{1}+\tau\right)-\lambda & 0 & 0 \\
\alpha_{1} & \left(\rho-\beta-\omega-\frac{\tau}{\kappa}\right)-\lambda & \mu \\
0 & \beta & -\mu-\lambda
\end{array}\right|=0(3.10)
\end{gathered}
$$

where $\lambda$ is the eigenvalue. Evaluating the determinant yields:

$$
\left(\alpha_{1}+\tau+\lambda\right)\left[\left(\left(\rho-\beta-\omega-\frac{\tau}{\kappa}\right)-\lambda\right)(\mu+\lambda)+\beta \mu\right]=0
$$

or

$$
\begin{gathered}
\left(\alpha_{1}+\tau+\lambda\right)\left[\left(-\lambda^{2}+\left(\rho-\beta-\omega-\frac{\tau}{\kappa}-\mu\right) \lambda+\right.\right. \\
\left.\left.\left(\rho-\omega-\frac{\tau}{\kappa}\right) \mu\right)\right]=0 .
\end{gathered}
$$

Solving equation (3.11) gives

$$
\begin{gathered}
\lambda_{1}=-\left(\alpha_{1}+\tau\right)<0, \\
\lambda_{2}=\frac{1}{2}\left(\rho-\beta-\omega-\frac{\tau}{\kappa}-\mu\right)-\frac{1}{2} \sqrt{\left(\rho-\beta-\omega-\frac{\tau}{\kappa}-\mu\right)^{2}+4 \mu\left(\rho-\omega-\frac{\tau}{\kappa}\right)}
\end{gathered}
$$

and

$$
\lambda_{3}=\frac{1}{2}\left(\rho-\beta-\omega-\frac{\tau}{\kappa}-\mu\right)+\frac{1}{2} \sqrt{\left(\rho-\beta-\omega-\frac{\tau}{\kappa}-\mu\right)^{2}+4 \mu\left(\rho-\omega-\frac{\tau}{\kappa}\right)} .
$$

Since

$$
\rho-\omega-\frac{\tau}{\kappa}<0
$$

then

$$
\left(\rho-\beta-\omega-\frac{\tau}{\kappa}-\mu\right)<0
$$

Therefore,

$$
\lambda_{2}<\frac{1}{2}\left(\rho-\beta-\omega-\frac{\tau}{\kappa}-\mu\right)-\frac{1}{2}\left(\rho-\beta-\omega-\frac{\tau}{\kappa}-\mu\right)=0 \Rightarrow \lambda_{2}<0,
$$

and

$$
\begin{gathered}
\lambda_{3}<\frac{1}{2}\left(\rho-\beta-\omega-\frac{\tau}{\kappa}-\mu\right)+\frac{1}{2}\left(\rho-\beta-\omega-\frac{\tau}{\kappa}-\mu\right)=(\rho-\beta- \\
\left.\omega-\frac{\tau}{\kappa}-\mu\right)<0 .
\end{gathered}
$$


Since all the eigenvalues are negative, we then conclude that the equilibrium state of the model is uniformly and asymptotically stable (UAS) provided $\frac{\|\boldsymbol{b}(t)\|}{|t|} \rightarrow 0$ as $|t| \rightarrow 0$, hence the proof.

\subsubsection{Stability Analysis of the Equilibrium State of the \\ Model Equations (Top-Down Processing)}

Similarly, the stability analysis of the equilibrium state of

$$
E_{2}=\left(\begin{array}{c}
\frac{\left[(\mu+v)\left(\rho-\beta-\omega-\frac{\tau}{\kappa}\right)+\beta \mu\right] I^{*}}{(\mu+v)\left(\alpha_{2}+\tau\right)\left(\rho-\beta-\omega-\frac{\tau}{\kappa}\right)+\beta\left[\mu\left(\alpha_{2}+\tau\right)+\alpha_{2} v\right]}, \frac{-\alpha_{2}(\mu+v) I^{*}}{(\mu+v)\left(\alpha_{2}+\tau\right)\left(\rho-\beta-\omega-\frac{\tau}{\kappa}\right)+\beta\left[\mu\left(\alpha_{2}+\tau\right)+\alpha_{2} v\right]}, \\
\frac{-\alpha_{2} \beta I^{*}}{(\mu+v)\left(\alpha_{2}+\tau\right)\left(\rho-\beta-\omega-\frac{\tau}{\kappa}\right)+\beta\left[\mu\left(\alpha_{2}+\tau\right)+\alpha_{2} v\right]}
\end{array}\right)
$$

of the system (3.4)-(3.6) is uniformly and asymptotically stable (UAS).

Proof:

Here,

$$
\begin{gathered}
A=\left(\begin{array}{ccc}
-\left(\alpha_{2}+\tau\right) & 0 & v \\
\alpha_{2} & \left(\rho-\beta-\omega-\frac{\tau}{\kappa}\right) & \mu \\
0 & \beta & -(\mu+v)
\end{array}\right), \\
y=\left(\begin{array}{l}
x_{2} \\
y_{2} \\
z_{2}
\end{array}\right) \text { and } b(t)=\left(\begin{array}{c}
I(t) \\
0 \\
0
\end{array}\right) .
\end{gathered}
$$

The eigenvalues are computed by solving

$$
\left|\begin{array}{ccc}
-\left(\alpha_{2}+\tau\right)-\lambda & |\mathrm{A}-\lambda \mathrm{I}|= \\
\alpha_{2} & 0 & u \\
0 & \left(\rho-\beta-\omega-\frac{\tau}{\kappa}\right)-\lambda & \mu \\
\beta & -(\mu+v)-\lambda
\end{array}\right|=0
$$

The corresponding characteristic equation is given as:

$$
\begin{gathered}
\lambda^{3}-\left[\left(\alpha_{2}+\tau\right)-(\mu+v)+\left(\rho-\beta-\omega-\frac{\tau}{\kappa}\right)\right] \lambda^{2} \\
-\left\{\begin{array}{c}
{\left[\left(\alpha_{2}+\tau\right)+(\mu+v)\right]\left(\rho-\beta-\omega-\frac{\tau}{\kappa}\right)} \\
+\left(\alpha_{2}+\tau\right)(\mu+v)+\beta \mu
\end{array}\right\} \lambda \\
-\left\{\begin{array}{c}
{\left[\left(\alpha_{2}+\tau\right)+(\mu+v)\right]\left(\rho-\beta-\omega-\frac{\tau}{\kappa}\right)} \\
+\beta \mu\left(\alpha_{2}+\tau\right)+\alpha_{2} \beta v
\end{array}\right\}=0
\end{gathered}
$$

To determine the sign of the roots of the characteristic equation (3.13), we use the Routh-Hurwitz criterion stated in theorem (3.2) namely

(i) $A_{0}, A_{1}, A_{2}>0$, and

(ii) $A_{2} A_{1}>A_{0}$.

Now,

$$
\begin{aligned}
& A_{2}=-\left[\left(\alpha_{2}+\tau\right)-(\mu+v)+\left(\rho-\beta-\omega-\frac{\tau}{\kappa}\right)\right] \\
& A_{1}=-\left\{\begin{array}{c}
{\left[\left(\alpha_{2}+\tau\right)+(\mu+v)\right]\left(\rho-\beta-\omega-\frac{\tau}{\kappa}\right)} \\
+\left(\alpha_{2}+\tau\right)(\mu+v)+\beta \mu
\end{array}\right\} \\
& A_{0}=-\left\{\begin{array}{c}
{\left[\left(\alpha_{2}+\tau\right)+(\mu+v)\right]\left(\rho-\beta-\omega-\frac{\tau}{\kappa}\right)} \\
+\beta \mu\left(\alpha_{2}+\tau\right)+\alpha_{2} \beta v
\end{array}\right\}
\end{aligned}
$$

the top-down processing model is presented in the theorem below:

\subsubsection{Theorem 3.4}

Given $\alpha_{2}, \beta, \mu, \tau, \rho, \omega, v \geq 0, \tau<\kappa$. If $\rho<\beta+\omega+\frac{\tau}{\kappa}$ and $I^{*} \neq 0$,

then the equilibrium state
But,

$$
\begin{array}{r}
A_{0}=-\left\{\left[\left(\alpha_{2}+\tau\right)+(\mu+v)\right]\left(\rho-\beta-\omega-\frac{\tau}{\kappa}\right)\right. \\
\left.+\beta \mu\left(\alpha_{2}+\tau\right)+\alpha_{2} \beta v\right\}>0
\end{array}
$$

if and only if

$$
\begin{gathered}
{\left[\left(\alpha_{2}+\tau\right)+(\mu+v)\right]\left(\rho-\beta-\omega-\frac{\tau}{\kappa}\right)+\beta \mu\left(\alpha_{2}+\tau\right)+} \\
\alpha_{2} \beta v<0 \\
\Rightarrow \rho+\frac{\left[\beta \mu\left(\alpha_{2}+\tau\right)+\alpha_{2} \beta v\right]}{\left[\left(\alpha_{2}+\tau\right)+(\mu+v)\right]}<\beta+\omega+\frac{\tau}{\kappa} \\
\Rightarrow \rho<\beta+\omega+\frac{\tau}{\kappa}
\end{gathered}
$$

Therefore by $(3.7)^{*} A_{0}>0$.

$$
\begin{gathered}
A_{1}=-\left\{\left[\left(\alpha_{2}+\tau\right)+(\mu+v)\right]\left(\rho-\beta-\omega-\frac{\tau}{\kappa}\right)\right. \\
\left.+\left(\alpha_{2}+\tau\right)(\mu+v)+\beta \mu\right\}>0
\end{gathered}
$$

if and only if

$$
\begin{gathered}
{\left[\left(\alpha_{2}+\tau\right)+(\mu+v)\right]\left(\rho-\beta-\omega-\frac{\tau}{\kappa}\right)+\left(\alpha_{2}+\tau\right)(\mu+v)} \\
+\beta \mu<0 \\
\Rightarrow \rho+\frac{\left[\left(\alpha_{2}+\tau\right)(\mu+v)+\beta \mu\right]}{\left[\left(\alpha_{2}+\tau\right)+(\mu+v)\right]}<\beta+\omega+\frac{\tau}{\kappa} \\
\Rightarrow \rho<\beta+\omega+\frac{\tau}{\kappa}
\end{gathered}
$$

Again, by $(3.7)^{*} A_{1}>0$.

$$
A_{2}=-\left[\left(\alpha_{2}+\tau\right)-(\mu+v)+\left(\rho-\beta-\omega-\frac{\tau}{\kappa}\right)\right]>0
$$

if and only if

$$
\begin{gathered}
{\left[\left(\alpha_{2}+\tau\right)-(\mu+v)+\left(\rho-\beta-\omega-\frac{\tau}{\kappa}\right)\right]<0} \\
\Rightarrow \rho+\left(\alpha_{2}+\tau\right)<\beta+\omega+\frac{\tau}{\kappa}+(\mu+v) \\
\Rightarrow \rho<\beta+\omega+\frac{\tau}{\kappa}+(\mu+v)
\end{gathered}
$$


Therefore, $A_{0}, A_{1}, A_{2}>0$.

Next, we show that $A_{2} A_{1}-A_{0}>0$

$$
\begin{array}{r}
A_{2} A_{1}-A_{0}=\left[\left(\alpha_{2}+\tau\right)-(\mu+v)+(\rho-\beta-\omega-\right. \\
\left.\left.\frac{\tau}{\kappa}\right)\right]\left\{\left[\left(\alpha_{2}+\tau\right)+(\mu+v)\right]\left(\rho-\beta-\omega-\frac{\tau}{\kappa}\right)+\left(\alpha_{2}+\tau\right)(\mu+\right. \\
v)+\beta \mu\}+\left\{\begin{array}{c}
{\left[\left(\alpha_{2}+\tau\right)+(\mu+v)\right]\left(\rho-\beta-\omega-\frac{\tau}{\kappa}\right)} \\
+\beta \mu\left(\alpha_{2}+\tau\right)+\alpha_{2} \beta v
\end{array}\right\}(3.17)
\end{array}
$$

It is established by (3.16) that

$$
\begin{gathered}
\mathrm{P}<\beta+\omega+\frac{\tau}{\kappa}+(\mu+v) \\
\Rightarrow \rho-(\mu+v)<\beta+\omega+\frac{\tau}{\kappa}
\end{gathered}
$$

Then, substituting the left hand side of (3.18) into (3.17) and changing the equality into an inequality, we have

$$
\begin{gathered}
A_{2} A_{1}-A_{0}>\left[\left(\alpha_{2}+\tau\right)-(\mu+v)+(\mu+v)\right]\left\{\left[\left(\alpha_{2}+\tau\right)+\right.\right. \\
\left.(\mu+v)](\mu+v)+\left(\alpha_{2}+\tau\right)(\mu+v)+\beta \mu\right\}+\left\{\left[\left(\alpha_{2}+\tau\right)+\right.\right. \\
\left.(\mu+v)](\mu+v)+\beta \mu\left(\alpha_{2}+\tau\right)+\alpha_{2} \beta v\right\} \\
=\left(\alpha_{2}+\tau\right)\left\{\left[\left(\alpha_{2}+\tau\right)+(\mu+v)\right](\mu+v)+\left(\alpha_{2}+\tau\right)(\mu+\right. \\
v)+\beta \mu\}+\left\{\left[\left(\alpha_{2}+\tau\right)+(\mu+v)\right](\mu+v)+\beta \mu\left(\alpha_{2}+\tau\right)+\right. \\
\left.\alpha_{2} \beta v\right\} \\
=\left[\left(\alpha_{2}+\tau\right)+(\mu+v)\right]\left(\alpha_{2}+\tau\right)(\mu+v)+\left(\alpha_{2}+\tau\right)^{2}(\mu+ \\
v)+\left(\alpha_{2}+\tau\right) \beta \mu+\left[\left(\alpha_{2}+\tau\right)+(\mu+v)\right](\mu+v)+ \\
\beta \mu\left(\alpha_{2}+\tau\right)+\alpha_{2} \beta v>0
\end{gathered}
$$

$A_{2} A_{1}-A_{0}>0 \Rightarrow A_{2} A_{1}>A_{0}$ as required.

Hence, the system (3.4) - (3.6) is uniformly and asymptotically stable (UAS).

\section{Numerical Results}

In these numerical experiments, we investigate the effects of attention, repetition and rehearsal on the learning process. Here, emphasis is being laid on the amount of information in the long-term memory. Therefore, we present the following experiments: (a) To examine the amount information in the long-term memory when either one of attention, repetition or rehearsal is zero for both mathematical models (bottom-up and top-down processing). (b) To determine the effects of repetition and rehearsal on the amount of information in the long-term memory for the two mathematical models. (c) To further determine the effects of repetition and rehearsal on the amount of information in the long-term memory for the two mathematical models.

The time axis $(t)$ is equally spaced with step size $h=0.5$ and the external stimulus function $I(t)$ is given as $I(t)=$ $t^{2}, t \in[0, \infty)$. A computer program is developed in MATLAB to solve the two models using the classical RungeKutta method of the fourth order for first order systems of differential equations. The plot of results generated for each numerical experiments are presented below:

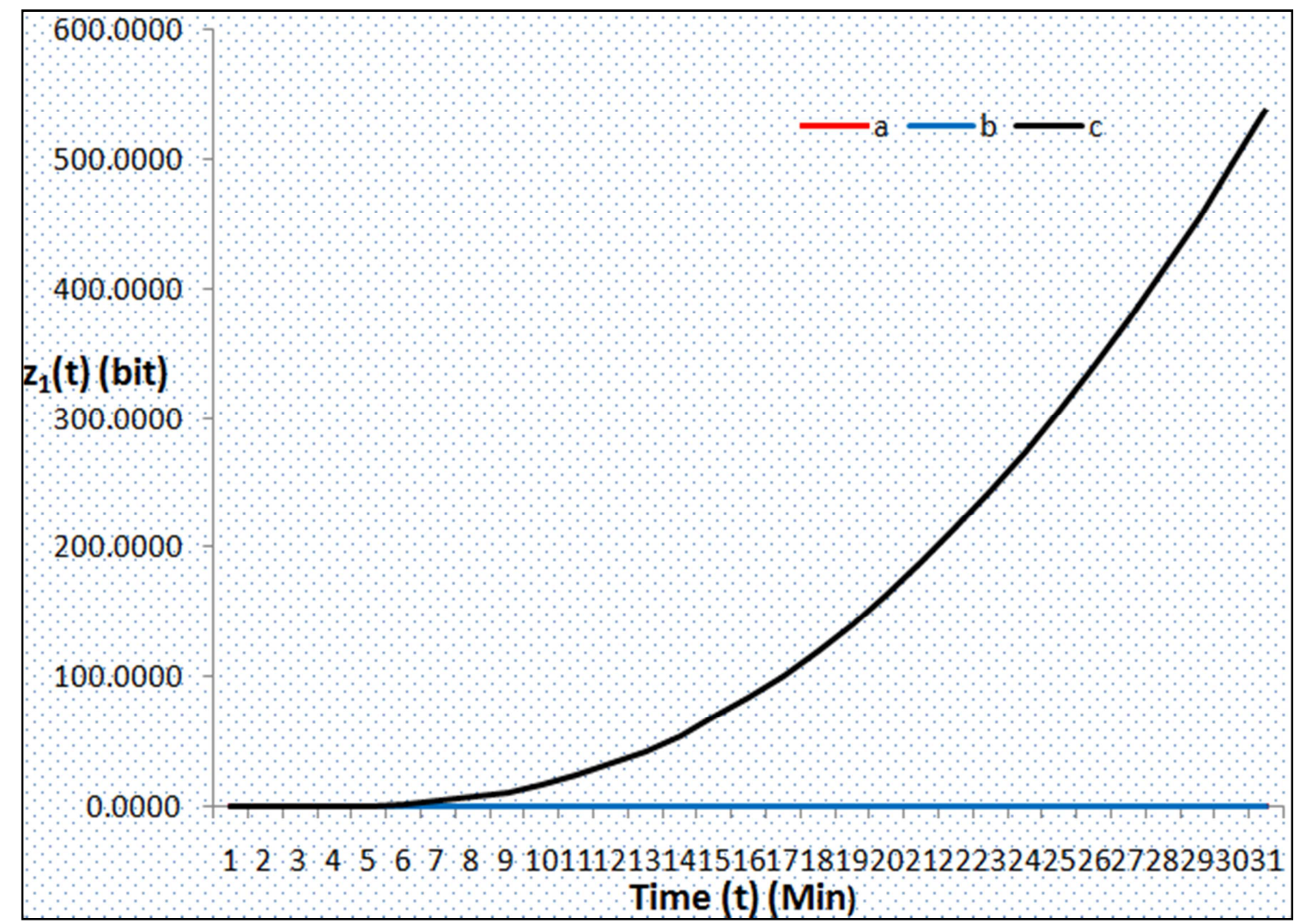

Figure 4. Graphs of Long-Term Memory $z_{1}(t)$ as a function of time $t$ in the following case: (a) when $\alpha_{1}=0.00, \rho=0.30, \beta=0.25$ (red) (b) when $\alpha_{1}=0.50$, $\rho=0.00, \beta=0.25$ (blue) (c) when $\alpha_{1}=0.50, \rho=0.30, \beta=0.00$ (black) using bottom-up processing model. Initial values: $x_{1}(0)=0, y_{1}(0)=$ 0 and $z_{1}(0)=0$. Other parameters remain fixed. 


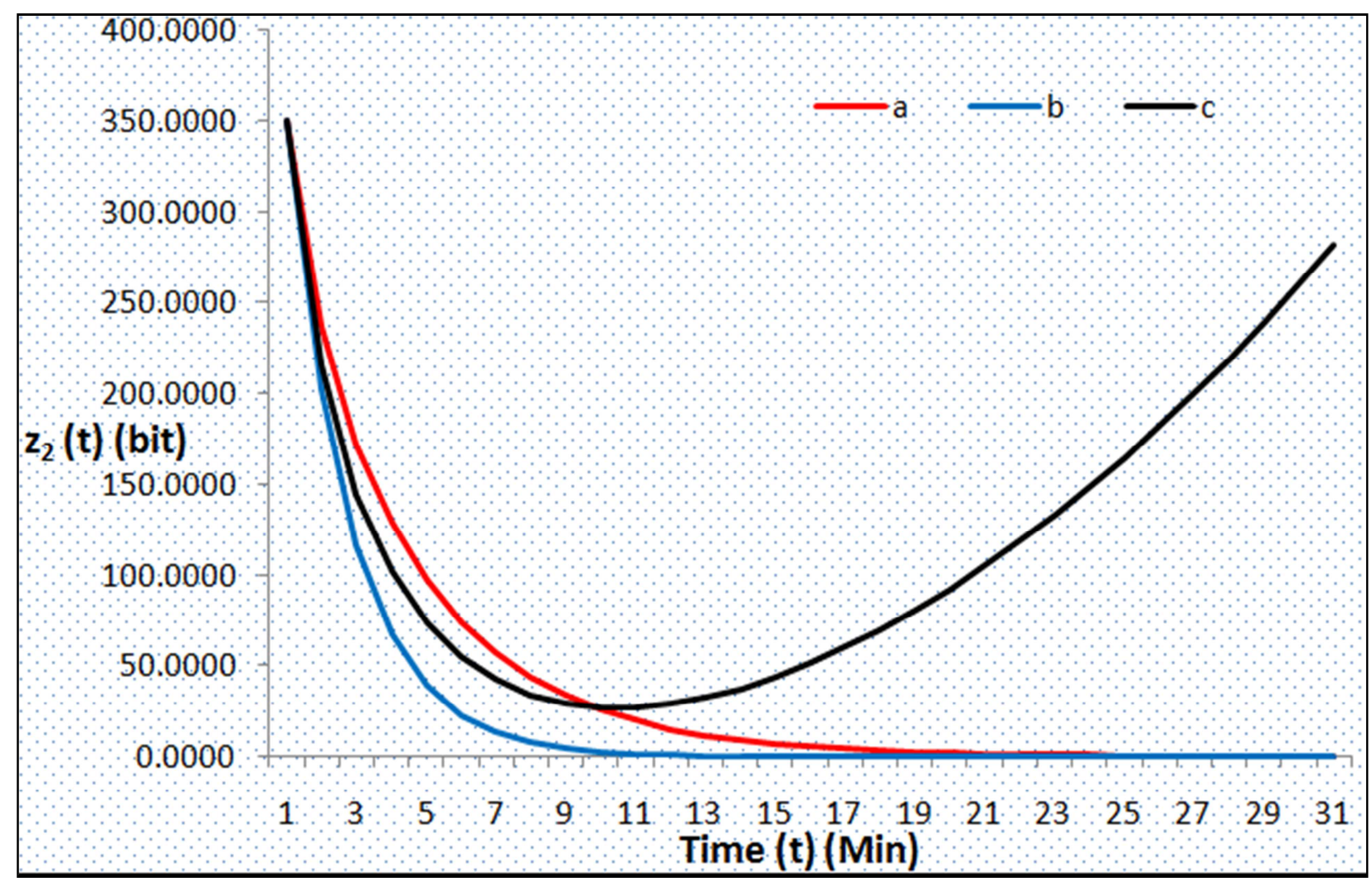

Figure 5. Graphs of Long-Term Memory $z_{2}(t)$ as a function of time $t$ in the following cases: (a) when $\alpha_{1}=0.00, \rho=0.30, \beta=0.25$ (red) (b) when $\alpha_{1}=$ $0.50, \rho=0.00, \beta=0.25$ (blue) (c) when $\alpha_{1}=0.50, \rho=0.30, \beta=0.00$ (black) using top-down processing model. Initial values: $x_{2}(0)=0, y_{2}(0)=$ 0 and $z_{2}(0)=350$. Other parameters remain fixed.

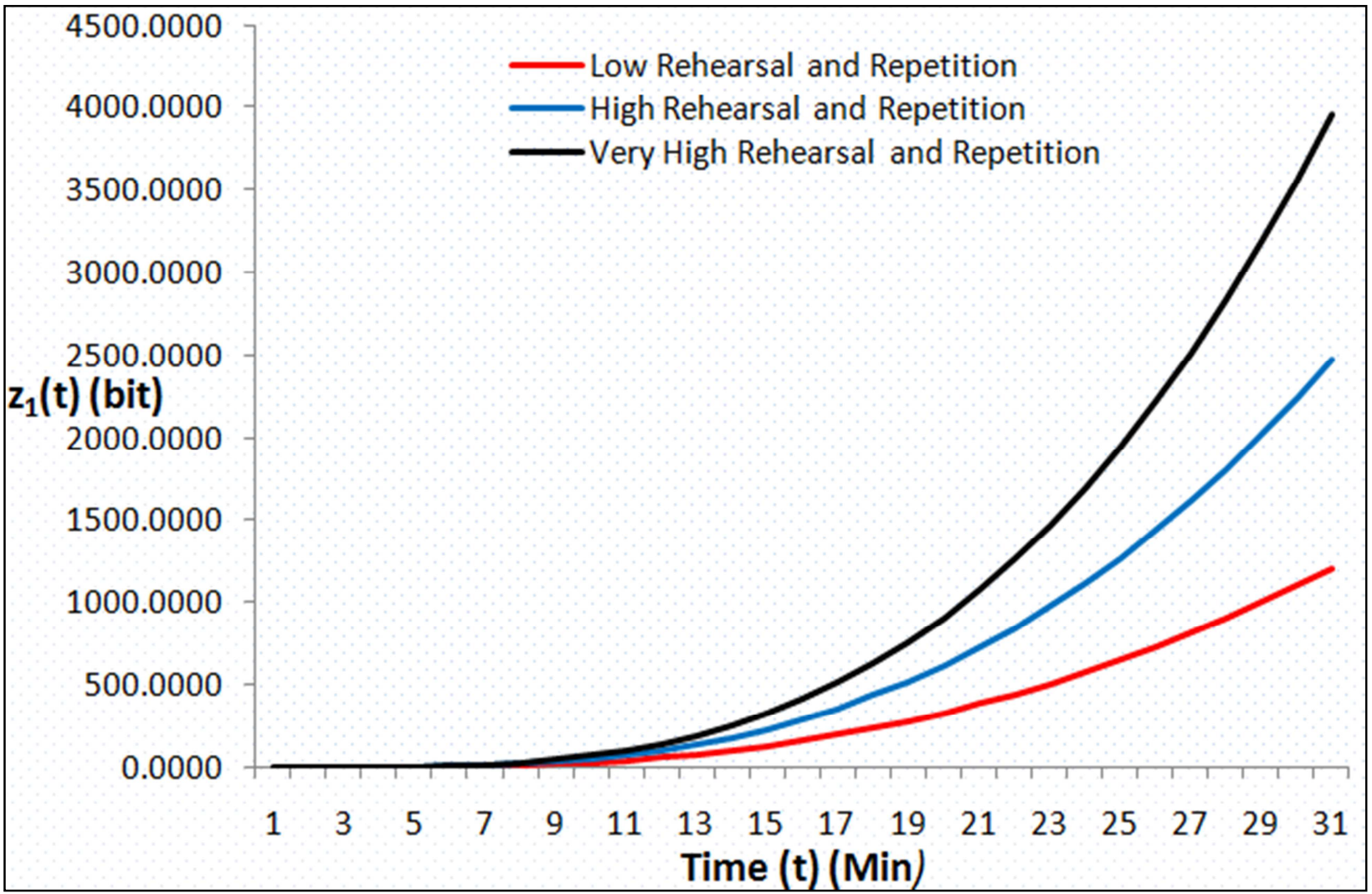

Figure 6. Graphs of Long-Term Memory $z_{1}(t)$ as a function of time $t$ in the following cases: (a) low rehearsal $(\beta=0.35)$ and repetition $(\rho=0.50)($ red $)(b)$ high rehearsal $(\beta=0.65)$ and repetition $(\rho=0.60)$ (blue) (c) very rehearsal $(\beta=0.95)$ and repetition $(\rho=0.70)$ (black) using bottom-up processing model. Initial values: $x_{1}(0)=0, y_{1}(0)=0$ and $z_{1}(0)=0$. Other parameters remain fixed. 


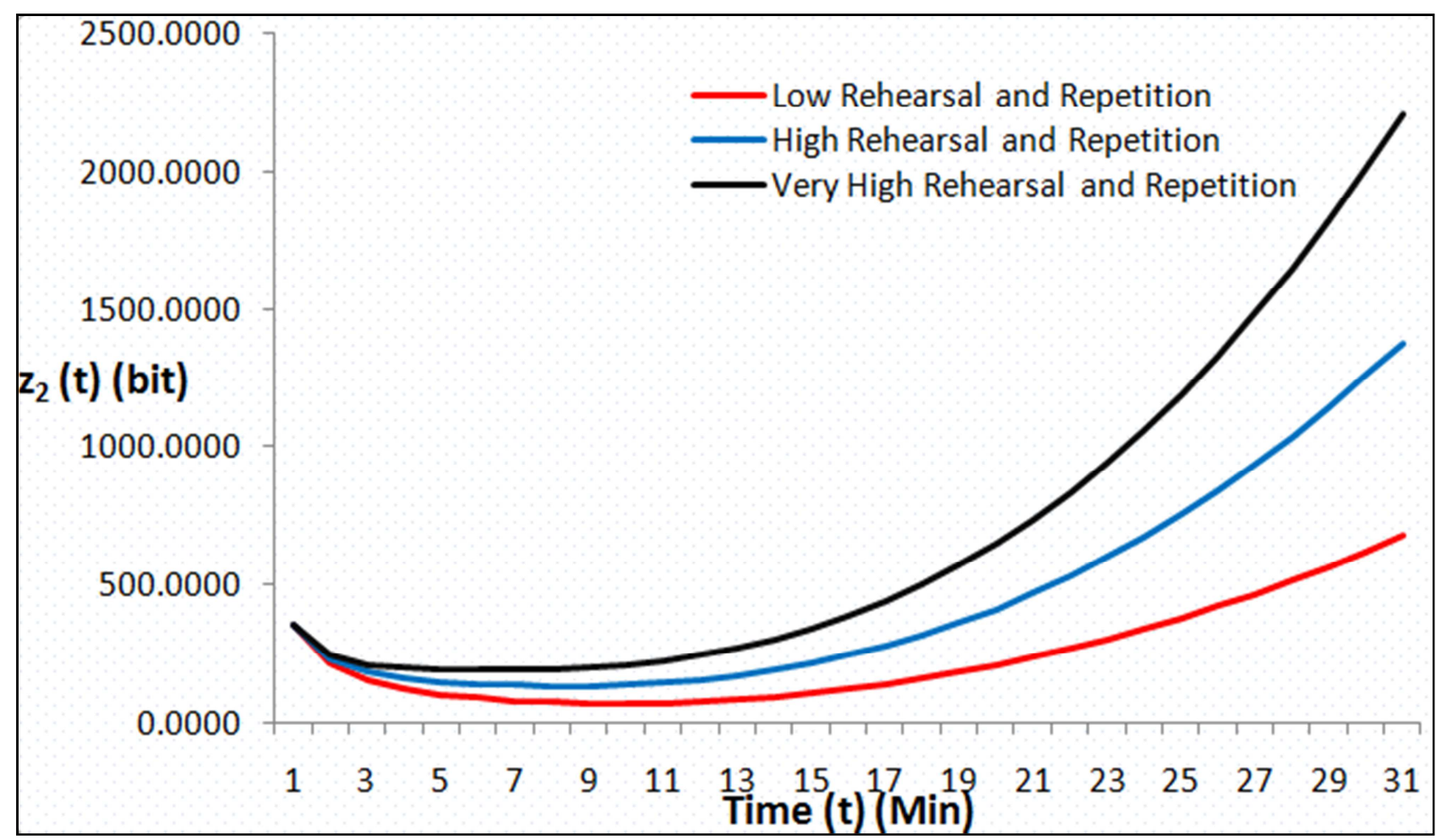

Figure 7. Graphs of Long-Term Memory $z_{2}(t)$ as a function of time $t$ in the following cases: (a) low rehearsal $(\beta=0.35)$ and repetition $(\rho=0.50)($ red $)(b)$ high rehearsal $(\beta=0.65)$ and repetition $(\rho=0.60)$ (blue) (c) very rehearsal $(\beta=0.95)$ and repetition $(\rho=0.70)$ (black) top-down processing model. Initial values: $x_{2}(0)=0, y_{2}(0)=0$ and $z_{2}(0)=350$. Other parameters remain fixed.

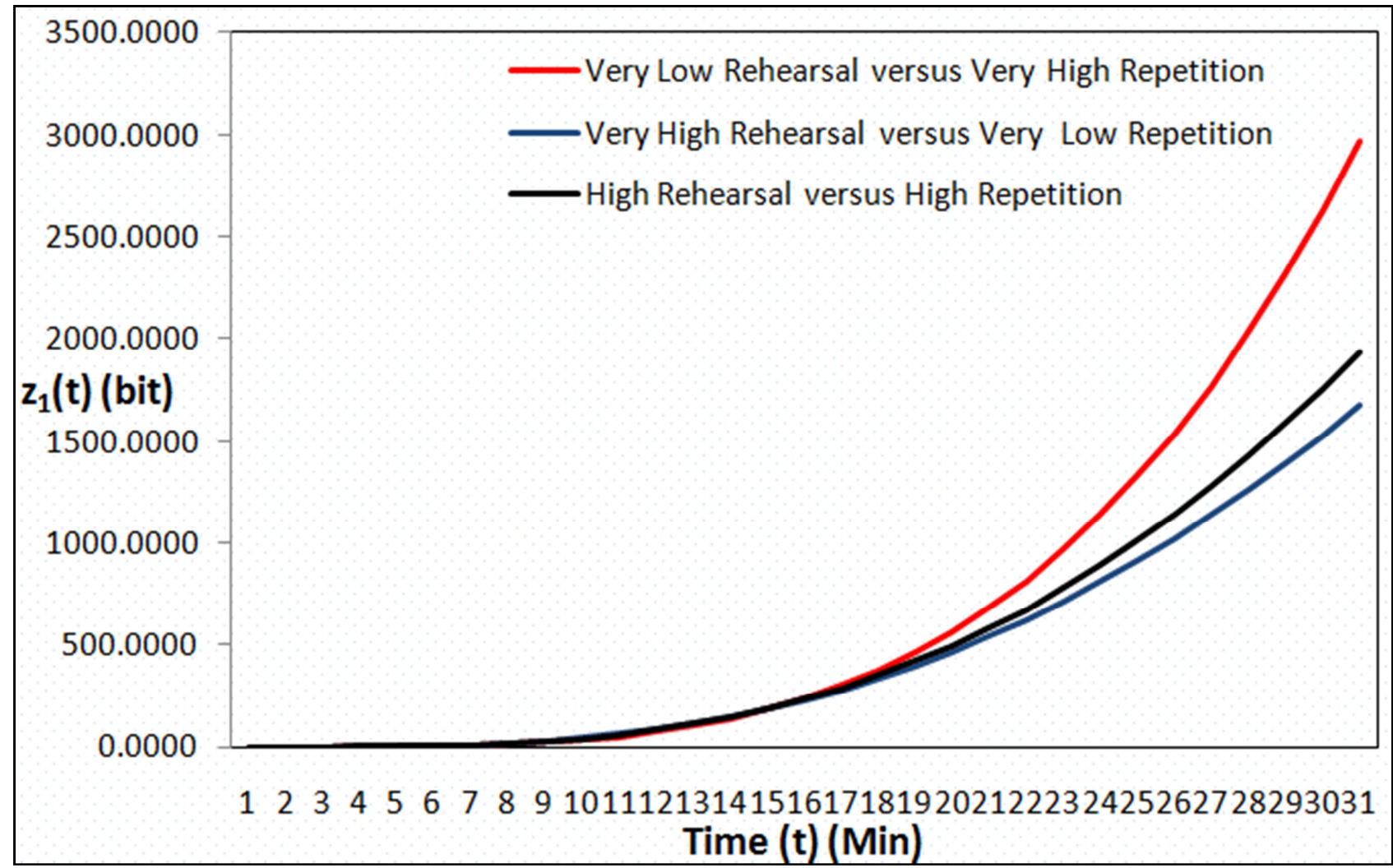

Figure 8. Graphs of Long-Term Memory $z_{1}(t)$ as a function of time $t$ in the following cases: (a) very low rehearsal $(\beta=0.25)$ versus very high repetition $(\rho=0.75)$ (red) (b) very high rehearsal $(\beta=0.75)$ versus very low repetition $(\rho=0.25)$ (blue) (c) high rehearsal $(\beta=0.50)$ versus high repetition $(\rho=$ 0.50 ) (black) using bottom-up processing model. Initial values: $x_{1}(0)=0, y_{1}(0)=0$ and $z_{1}(0)=0$.Other parameters remain fixed. 


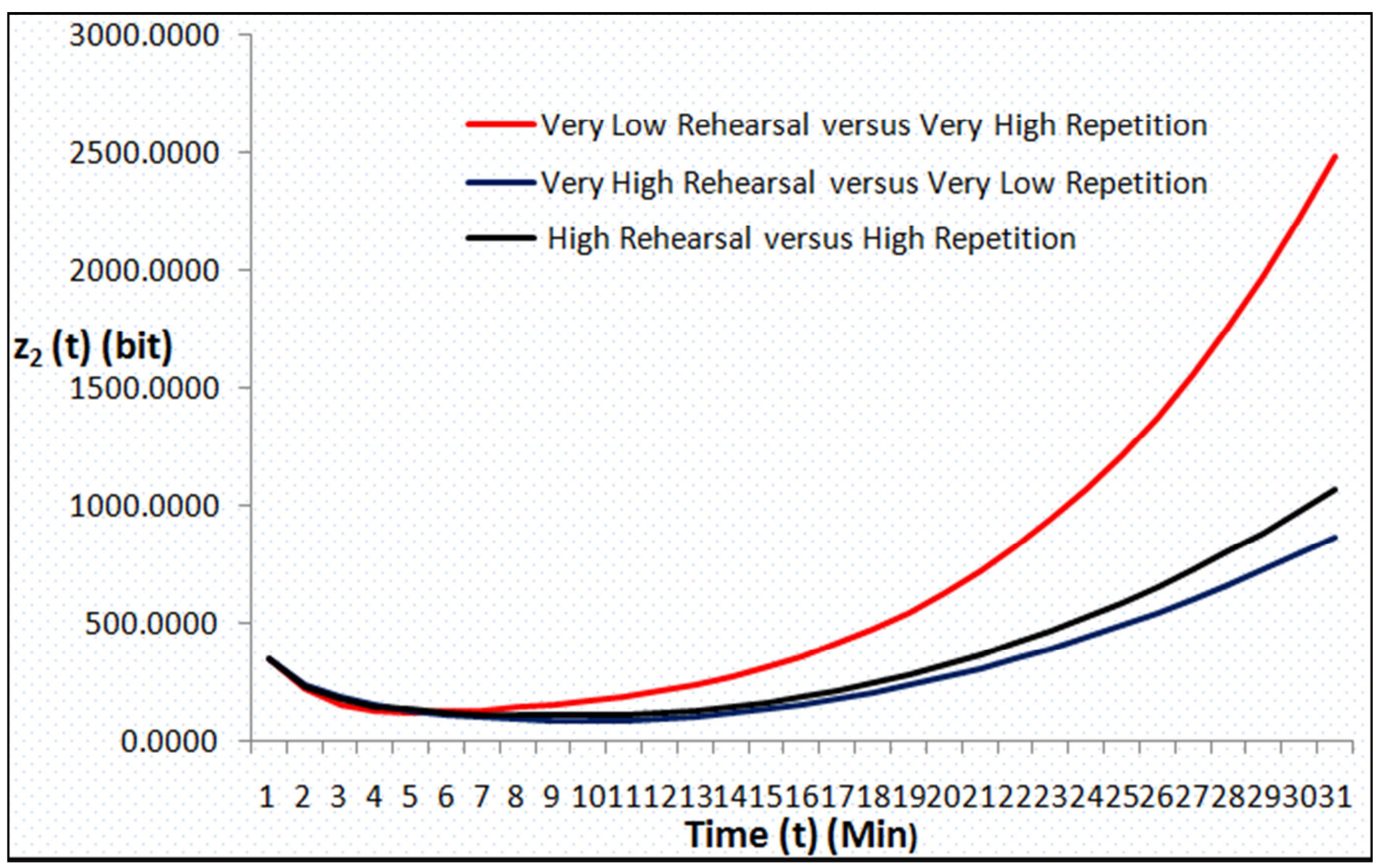

Figure 9. Graphs of Long-Term Memory $z_{1}(t)$ as a function of time $t$ in the following cases: (a) very low rehearsal $(\beta=0.25)$ versus very high repetition $(\rho=0.75)$ (red) (b) very high rehearsal $(\beta=0.75)$ versus very low repetition $(\rho=0.25)$ (blue) (c) high rehearsal $(\beta=0.50)$ versus high repetition $(\rho=$ 0.50 ) (black) using top-down processing model. Initial values: $x_{2}(0)=0, y_{2}(0)=0$ and $z_{2}(0)=350$.Other parameters remain fixed.

\section{Results Discussion}

\subsection{Introduction}

In this chapter, we discuss the results of the numerical experiments carried out. The plots of these results are shown in Figures (4)-(9).

\subsection{Discussions of the Numerical Results of the Model Equations}

In total, three (3) sets of numerical experiments were performed. The overall aim of these experiments is to determine the effects of attention, repetition and rehearsal on the flow of information into the long term memory. From our results, we noted the following:

\subsubsection{Experiment One (A)}

Here, we investigate a situation whereby fresh information is introduced to an individual under the following conditions: (a) $\alpha_{1}=0.00, \rho=0.30, \beta=0.25$ (b) $\alpha_{1}=0.50, \rho=0.00$, $\beta=0.25$ and (c) $\alpha_{1}=0.50, \rho=0.30, \beta=0.00$ using bottom-up processing model as in Figure 4. We see that, the information retained in the Long-Term Memory (LTM) increases with time only in case (c). This means that attention and rehearsal are very important in retaining fresh information in the Long-Term Memory (LTM).

\subsubsection{Experiment One (B)}

In this experiment, we investigate a similar situation for an individual already having prior knowledge of the information being passed, as follows: (a) $\alpha_{1}=0.00, \rho=0.30, \beta=0.25$ (b) $\alpha_{1}=0.50, \rho=0.00, \beta=0.25$ and (c) $\alpha_{1}=0.50$, $\rho=0.30, \beta=0.00$. The result here (Figure 5) is consistent with that of the previous case. That is, that attention and rehearsal are very important in retaining information in the Long-Term Memory (LTM) even for someone with prior knowledge but that, in the absence of repetition the prior information declines as shown by the graph in black colour.

\subsubsection{Experiment Two (A)}

In this experiment, we determined the effect of rehearsal combine with repetition in direct variation for an inexperienced individual, the graph with black colour (very high rehearsal and repetition) in Figure 6 illustrated that, very high rehearsal and repetition are very useful.

\subsubsection{Experiment Two (B)}

Here, we examine a similar scenario with Experiment Two (A) for an individual with prior information and the two results are consistent. It is also observed that the information obtained for an individual with fresh knowledge supersedes that of the individual with prior knowledge under the same condition of rehearsal and repetition due to the effect automaticity.

\subsubsection{Experiment Three (A)}

In this case, we further seek to determine the effect of rehearsal together with repetition in an inverse variation for an inexperienced individual. The graph with red colour in Figure 8 shows the best combination. It means that repetition maintains or refreshes information in Short-Term Memory (STM) for a long time which gives opportunity for effective transfer of information to the Long-Term Memory (LTM). 


\subsubsection{Experiment Three (B)}

This experiment is very similar to $3 \mathrm{~A}$ above, however, it now considered an experience driven processing. The red curve in Figure 9 further demonstrated that, an increasing repetition keeps fresh a piece of information in the ShortTerm Memory (STM) literally called the mind thereby making rehearsal effective.

\section{Conclusion}

In this research, we transformed the stage model developed by [2], comprehensively presented by [7] into two deterministic mathematical models. The first evolved from data driven processing (bottom-up processing) while the second came from experience driven processing (top-down processing). The model equations are formulated with the aid of schematic diagrams in Figures (2) and (3) which evolved from the stage model (Figure 1). The equilibrium states of the models were found to be uniformly and asymptotically stable under given conditions on the models parameters.

We studied the dependence of the information processing dynamics of a human brain on attention, repetition and rehearsal. Other parameters were held fixed in each set of experiments to investigate clearly the efficacy of the parameter(s) under examination. Hypothetical values were assigned to the model parameters and a finite difference scheme was used in the numerical experiments. Numerical experiments, using hypothetical data further determined the role played by attention, rehearsal and repetition on the human brain information processing dynamics. We found that, adequate attention should be given to information in the learning process. It is also important to refresh the mind with information by constant rehearsal. Furthermore, repetition is also recommended as an effective way of retaining information.

\section{References}

[1] Abbot B. (2002). Human memory. Fort Wayne: Indiana University-Purdue University at Fort Wayne, Psychology Department. 1-20. http://users.ipfw.edu/abbot/120/LongTermMemory.html.

[2] Atkinson, R. and Shiffrin, R. (1968). Human memory. A proposed system and its control processes. In K. Spence \& J. Spence (Eds.), The psychology of learning and motivation. Princeton, NJ: Van Nostrand. pp. 1-5.
[3] Driscoll, M. (2001). Psychology of learning for assessment (2nd ed). Boston: Allyn and Bacon. p. 81.

[4] Gantmacher, F. R. (1964). Matrix Theory. Vol. II Chelsea Publishing Company, New York. p. 374.

[5] Gibson, J. (1979). The ecological approach to visual perception. Boston: Houghton Mifflin. 332pp.

[6] Grimshaw, R.. (1990). Nonlinear ordinary differential equations. Pi-Square Press, Nottingham. pp. 23-44.

[7] Huitt, W., (2000). The information processing approach. Educational Psychology Interactive. Valdosta, GA: Valdosta State University. $\quad$ pp. 12 . http://chiron.valdosta.edu/whuitt/col/cogsys/infoproc.html.

[8] Jeffrey, D. S., Martin, P. and Geoffrey, F. W. (2007). Commented on top-down verses bottom up control of attention in the prefrontal and posterior parietal cortices. PubMed 44, www.sciencemag.org.

[9] Lin P. H, Luck S. J. (2009). The Influence of Similarity on Visual Working Memory Representations. Visual Cognition. 17(3):356-372.

[10] Miller, G., Galanter E. and Pribram, K. (1960). Plans and the structure of behavior. New York: Holt, Rinehart, \& Winston. 226pp.

[11] Rosenholtz, R, Jie, H. and Krista, A. E (2012). Rethinking the role of top-down attention in vision effects attributable to a lossy representation in peripheral vision. PMC3272623. http://www.ncbi.nlm.nih.gov/pmc/articles/PMC3272623/.

[12] Sousa, D. (2001). How the Brain Learns. Thousand Oaks, California: Corwin Press. pp. 127 -175. http://www.pitt.edu/ suthers/infsci1042/attention.html.

[13] Stacey, T. L. and William, G., (2003). Information Processing and Memory: Theory and Applications. pp. 2-4. http://www.chiron.valdosta.edu.

[14] Suthers, D. (1996). Attention and automaticity. Pittsburgh: University of Pittsburg, Learning Research and Development Center. 1-10. http://www.pitt.edu/ suthers/infsci1042/attention.html.

[15] Wei Z, Wang X. J, Wang H. D. (2012). From Distributed Resources to Limited Slots in Multiple-Item Working Memory: A Spiking Network Model with Normalization. The Journal of Neuroscience.32 (33):11228-11240.

[16] Zekveld, A. A., Heslenfeld, D. J, Festen, J. M. and Schoonhoven, R. (2006). The top-down and bottom-up processes in speech comprehension. Neuroimage. 32(4):182636. http://journals.ohiolink.edu/. 